\title{
Hydrogels with Dual Thermo-Responsive Mechanical Performance
}

Hui Guo, $\uparrow$ Cécile Mussault, $\uparrow$ Alba Marcellan, Dominique Hourdet, Nicolas Sanson*

H. Guo, C. Mussault, A. Marcellan, D. Hourdet, N. Sanson

Soft Matter Sciences and Engineering

ESPCI Paris, PSL Research University, CNRS UMR 7615, 10 rue Vauquelin, F-75231, Paris cedex 05, France

UPMC Univ Paris 06, Sorbonne-Universités, 10 rue Vauquelin, F-75231 Paris cedex 05,

France

E-mail: nicolas.sanson@espci.fr

${ }^{\dagger}$ H. Guo and C. Mussault contributed equally to this work

\begin{abstract}
Dual thermo-responsive chemical hydrogels, combining poly( $N$-isopropylacrylamide $)$ (PNIPAm) side-chains within a poly( $N$-acryloylglycinamide) (PNAGA) network, were designed following a simple and versatile procedure. These hydrogels exhibit two phase transitions both at low (upper critical solution temperature, UCST) and high (lower critical solution temperature, LCST) temperatures thereby modifying their swelling, rheological and mechanical properties. These novel thermo-schizophrenic hydrogels pave the way for the development of thermo-toughening wet materials in a broad range of temperatures.
\end{abstract}




\section{Introduction}

Benefitting from their good biocompatibility, hydrogels have experienced rapid progress during the last two decades. ${ }^{[1]}$ Despite their wide potential in biomedicine and industry, ${ }^{[2,3]}$ applications of these aqueous-based soft materials have long been restricted due to their intrinsic poor mechanical strength, mainly related to the high hydration level and low efficiency of dissipation process. Since the last 15 years, different efficient strategies have been developed to improve their mechanical properties with either introducing inorganic charges forming nanocomposites or hybrid hydrogels ${ }^{[4,5]}$ or using smart macromolecular architectures such as double network systems, ${ }^{[6]}$ slide-ring gels, ${ }^{[7]}$ or tetra-PEG. ${ }^{[8]}$

During the past few years, another mode of hydrogel reinforcement based on the universal concept of polymer phase separation has been developed. For instance, Sun et al. elaborated chemical polyampholyte hydrogels which demonstrate high toughness and viscoelasticity. ${ }^{[9]}$ This increase of the mechanical properties was driven by the formation of phase-separated polymer domains, induced by electrostatic interactions between repeat units of opposite charge, playing the role of a highly dissipative viscoelastic network. However, the formation of these phase-separated domains requires a high concentration of polymer ranging between 30 and $50 \mathrm{wt} \%$. This concept of polymer phase separation can be also induced by adding a poor solvent of the macromolecular network. As shown by Sato et al., the tensile performance of polyacrylamide (PAM) gels can be greatly enhanced using water $/ N, N$ dimethylformamide (DMF) mixtures around 30/70\% in weight. ${ }^{[10]}$ On the other hand, the addition of a poor solvent leads to a strong deswelling of PAM gel reaching polymer concentration up to $56 \mathrm{wt} \%$. Besides, for specific applications, it could be also interesting to modify in a controlled manner the properties of hydrogels by applying external stimuli, such

as $\mathrm{pH}$ or temperature. ${ }^{[11-14]}$ Such behavior can be achieved by using thermoresponsive polymers and this was clearly demonstrated with the pioneering work of Shibayama on 
poly( $N$-isopropylacrylamide) (PNIPAm) hydrogels leading to an enhancement of the mechanical properties above their critical temperature. ${ }^{[15]}$ However, in most of the research works cited above, the polymer phase separation which is driving the mechanical reinforcement, is also responsible for a large volume transition that leads to highly concentrated macromolecular networks in a collapsed state (solvent content below $50 \mathrm{wt} \%$ ). Considering mechanical properties, this generally makes difficult to clearly dissociate the contribution related to the formation of a phase separated morphology from the one related to the increase of polymer concentration induced by the volume phase transition. In order to finely investigate the impact of the polymer phase separation on mechanical properties while avoiding the collapse of the gel with the formation of a weakly swollen network, we recently designed thermoresponsive cross-linked gels with different topologies. These hydrogels can retain a high level of water (more than $80 \mathrm{wt} \%$ ) on both sides of the transition temperature. ${ }^{[16 \text {, }}$ 17] To achieve this, lower critical solution temperature (LCST) polymer chains such as PNIPAm and hydrophilic poly( $N, N$-dimethylacrylamide) (PDMA) were combined into the same cross-linked architecture. Within this network, the hydrophilic counterpart allows to maintain a high level of hydration, even above the phase transition temperature of the PNIPAm. Using these hydrogels in the preparation state, it was possible to keep their volume constant, with a relatively high swelling state both well above and below the critical temperature and to clearly identify the role of the PNIPAm phase separation in the mechanical reinforcement. The designed hydrogels exhibit a strong thermo-toughening with excellent fatigue resistance, full recovery and remarkable fracture properties. So far, only LCST-type polymers have been investigated restricting both the nature and the range of applications.

As mentioned previously, the development of thermo-responsive hydrogels working in a broad range of temperatures, with a control of their properties induced either by heating and/or cooling, is an important requirement in order to extend the spectrum of applications. Very few examples of hydrogels exhibiting double thermo-responsive behavior in pure water, 
i.e, both an UCST and a LCST, were highlighted in the literature. Liu et al. elaborated hydrogels with a thermo-responsive behavior at low and high temperature by introducing poly(ethylene glycol) chains in PNIPAm network physically crosslinked with clay particles. ${ }^{[18]}$ This double temperature response is driven by an absorption competition between clay particles and both PEG and PNIPAm chains modifying the polymer chains conformation in the network at low and high temperatures. Hydrogels with opposite temperature behavior were also elaborated using a single copolymer constituted by ethylene oxide oligomonomers of different lengths, i.e., di(ethylene oxide) $\left(\mathrm{EO}_{2}\right)$ and oligo(ethylene oxide) $\left(\mathrm{EO}_{8-9}\right)$ physically crosslinked with clay particles. ${ }^{[19,20]}$ The transition temperature of this copolymer, analogue to the poly(ethylene oxide) (PEO) and representing an alternative to PNIPAm, can be tuned in a broad range of temperature by changing the ratio of ethylene oxide oligomonomers. The authors claim that the UCST and LCST behavior is due to the formation of loose and dense polymer/clay aggregates at low and high temperature respectively. Also, Yang et al. prepared chemical crosslinked hydrogels by copolymerizing vinylcaprolactam and zwitterionic monomers, known to their thermo-responsive behavior in linear polymer chains, into the same network. $^{[21]}$ However, These synthesized hydrogels present a very low response to the temperature compared to analogous copolymers. In the few examples listed, neither deeply investigate the temperature-induced mechanical properties.

Recently, neutral polymers exhibiting an upper critical solution temperature (UCST) in water, ${ }^{[22-25]}$ especially poly( $N$-acryloylglycinamide) (PNAGA), have received emerging concern. ${ }^{[26-28]}$ The phase transition process of PNAGA in water is driven at low temperature by intra- and intermolecular hydrogen bonding between repeat units located on either the same or different polymer chains. In the present work, we developed an original dual thermoresponsive hydrogel, combining LCST- and UCST-type polymers that will be able to thermally respond in isochoric conditions and to tune its mechanical properties over a broad range of temperature (Figure 1). Indeed, uniting PNAGA and PNIPAm into the same network, 
gives us a unique opportunity to investigate and compare the impact of antagonistic phase separation mechanisms, triggered at low and high temperatures, over the mechanical properties. Based on the polymer phase separation concept, the versatility of the designed hydrogels will provide a promising method to test the universality of the phase-separationinduced reinforcement.

\section{Experimental Section}

Detailed experimental section including used materials, characterization techniques, synthesis of NAGA monomer and hydrogels can be found in Supporting Information.

\section{Results and Discussion}

NAGA monomer was synthesized following a new procedure based on the synthesis of Seuring et al. but modified in order to both facilitate the synthesis process and avoid the presence of ionic groups, detrimental for the UCST behavior of PNAGA in water (Figure 1a). ${ }^{[29]}$ Indeed, by using only miscible organic solvents, this new synthesis procedure is less time-consuming by reducing the number of purification steps and avoiding the presence of ionic group (see procedure of NAGA synthesis, Figures S1-S3 and Table S1, Supporting Information). Dual thermo-responsive hydrogels were designed by copolymerizing equal amounts of NAGA monomer and PNIPAm macromonomer with additional chemical crosslinker. The resulting gel was named PAN where A and N stand for NAGA monomer and PNIPAm macromonomer, respectively. The dual thermo-responsive behavior of PAN 
hydrogel was finely investigated by turbidity experiments, swelling measurements, differential scanning calorimetry (DSC) and ${ }^{1} \mathrm{H}$ NMR spectroscopy as shown in Figure 2 and Figure S4 in Supporting Information. Owing to the combination of PNAGA and PNIPAm in the same network, turbidity experiments clearly highlight the dual thermo-response of PAN hydrogel in water with both UCST- and LCST-type behaviors (Figure 2a). Indeed, on both sides of ambient temperature, PAN hydrogel becomes increasingly turbid when the gel is either heated or cooled, indicating the phase separation process of PNIPAm and PNAGA, respectively (inset of Figure 2a). While no notable hysteresis was observed for the PNIPAm phase separation, the phase separation of PNAGA clearly exhibits a hysteresis of $10{ }^{\circ} \mathrm{C}$, even at low heating/cooling rates of $0.5{ }^{\circ} \mathrm{C} \min ^{-1}$, demonstrating a very slow kinetic process as already reported in the literature. ${ }^{[29]}$ According to the turbidity experiments, UCST- and LCST-type cloud points of PAN hydrogel were found to be 14 and $36{ }^{\circ} \mathrm{C}$ respectively in agreement with DSC experiments (Figure S4, Supporting Information). Taking into account the phase transition temperatures of the two polymers, the swelling ratio at equilibrium in pure water, $Q_{\mathrm{e}}$, was investigated as a function of temperature (Figure $2 \mathrm{~b}$ ). From 30 to $35^{\circ} \mathrm{C}$, PAN hydrogel swells $\left(Q_{\mathrm{e}}>10\right)$ as PNIPAm and PNAGA chains exhibit a coil conformation thereby fostering their hydrogen bonds with water molecules, which is in agreement with the transparency of PAN hydrogel (Figure 2a). By contrast, due to the UCST and LCST behaviors of the designed PAN hydrogel, NAGA (respectively NIPAm) polymer chains undergo a conformational transition at low (respectively high) temperature generating different polymer phase-separated domains depending on the gel topology (Figure 1). ${ }^{[16,17]}$ Despite the phase separation process, PAN hydrogel retains almost the same swelling ratio than in the preparation state both at high $\left(\mathrm{T} \geq 40{ }^{\circ} \mathrm{C}, Q_{\mathrm{e}} \cong Q_{0}=6\right)$ and low $\left(T=5{ }^{\circ} \mathrm{C}, Q_{\mathrm{e}} \cong 7\right)$ temperatures. This is mainly due to the osmotic contribution of hydrophilic polymer chains, namely PNIPAm at low temperature and PNAGA at high temperature, that promote the gel swelling or at least compensate the large collapse of the antagonistic polymer phase beyond 
the critical temperature (Figure S5, Supporting Information). Note that the phase transition of PNAGA in both pure PNAGA and PAN gels takes place in a broad range of temperatures as reported in the literature ${ }^{[30]}$ whereas a sharp transition is observed for PNIPAm based gels. As the equilibrium swelling ratios of PAN gels $\left(Q_{\mathrm{e}}\right)$ are always equal or higher than $Q_{0}$ in the whole range of temperatures, mechanical experiments can be carried out under isochoric conditions (without any risk of volume change) by simply working with gel samples maintained in their preparation state by immersion into immiscible solvent like paraffin oil for instance. Finally, the phase transition of both polymers was also evidenced from ${ }^{1} \mathrm{H}$ NMR measurements performed at different temperatures as shown in Figure 2c. Upon heating the NMR signals corresponding to methyl and methine groups of PNIPAm, respectively observed at 1.1 and $3.9 \mathrm{ppm}$ (zoom in Figure 2c), progressively decrease whereas the NMR signals of NAGA (3.5-4 ppm) exhibit a better resolution at $50{ }^{\circ} \mathrm{C}$. This set of experimental techniques clearly demonstrates the dual thermo-responsive behavior of PAN hydrogels in water.

As illustrated in Figure 3, the temperature phase separation of each thermo-responsive polymers, PNAGA and PNIPAm, strongly influences the viscoelastic properties of PAN hydrogel at low and high temperature respectively. Upon heating from 5 to $36^{\circ} \mathrm{C}$, the elastic modulus, $G^{\prime}$, first decreases continuously, from 7 to $5 \mathrm{kPa}$ and subsequently increases sharply until a value of $10 \mathrm{kPa}$ with increasing the temperature from 36 to $60{ }^{\circ} \mathrm{C}$. The increase of the elastic modulus on both sides of the minimum observed at $36^{\circ} \mathrm{C}\left(G^{\prime}=5 \mathrm{kPa}\right)$ is in good agreement with the phase separation process of the two thermo-responsive components of PAN gel and mirrors the previous turbidity and swelling experiments. Below the UCST-, and above the LCST-type cloud points, PNAGA and PNIPAm chains self-associate respectively into collapsed domains forming additional interactions and physical crosslinks into the polymer network. This dual thermo-reinforcement constitutes a major step compared to existing single thermo-reinforcement system in which thermo-responsive polymers were 
embedded into a hydrophilic matrix (Figure S6, Supporting Information). Compared to the UCST phase transition of PNAGA, the thermo-reinforcement induced by the LCST phase transition of PNIPAm appears more effective in term of elastic contribution and sharpness of the transition, which can be related either to different self-association mechanisms and/or kinetics effects. From these results, it appears obvious that mechanical properties can be effectively increased with temperature by modulating the chains solubility (from coil to globule conformation) and inducing collapsed domains into the grafted hydrogel. ${ }^{[16,17]}$ When the same experiment is carried out by cooling, from 50 to $5^{\circ} \mathrm{C}$, a similar behavior can be observed with a shift of the transition temperature from 10 to $15^{\circ} \mathrm{C}$ towards low temperatures mainly due to kinetic effects related to the thickness of the gel sample and to the relative high rate of cooling $\left(2^{\circ} \mathrm{C} \mathrm{min}^{-1}\right)$ compared to the slow kinetics of PNAGA phase separation. ${ }^{[29]}$

With the aim of getting a better overview of the impact of the dual temperature phase separation process on the mechanical performances, large strain behavior in tension mode was also investigated on PAN gel at various temperatures. As illustrated in Figure 4, PAN gel demonstrates a very clear temperature dependence under stretching. At $32{ }^{\circ} \mathrm{C}$, the initial tensile modulus, E, reaches a value of about $8 \mathrm{kPa}$ with a strain at break of $300 \%$ (Figure $4 \mathrm{a}$ ). In this condition, both UCST- and LCST-type associative interactions are expected to be ineffective and the gel response should mainly originate from entropic elasticity of the covalent network. At lower temperature $\left(T=5^{\circ} \mathrm{C}\right)$, the PNAGA phase separation improves the gel stiffness as shown by the value of the initial modulus around $E \sim 14 \mathrm{kPa}$ but slightly decreases at the same time its extensibility (150-180\%). Interestingly, above the phase separation of PNIPAm $\left(T=60^{\circ} \mathrm{C}\right)$, the self-association of PNIPAm side-chains simultaneously enhance both stiffness $(E \sim 32 \mathrm{kPa})$ and elongation at break (more than 1000\%). A simple estimate of the work of extension, given by the area under the tensile curve, yields $W \cong 270 \mathrm{~kJ}$ $\mathrm{m}^{-3}$ (compared to $W \cong 19 \mathrm{~kJ} \mathrm{~m}^{-3}$ at $32{ }^{\circ} \mathrm{C}$ ) revealing that physical interactions efficiently delay 
fracture. It is worth mentioning that the elastic modulus determined by mechanical testing are in agreement with those obtained from dynamic experiments, assuming pure incompressibility with $E=3 G$. The temperature dependence of the phase separation-induced thermo-toughness of PAN gel is explicitly shown in Figure $4 \mathrm{~b}$ where the variation of the initial tensile modulus is in good agreement with the expected phase transition temperatures of PNAGA $\left(T<10{ }^{\circ} \mathrm{C}\right.$, $E \sim 15-20 \mathrm{kPa})$ and PNIPAm $\left(T>35^{\circ} \mathrm{C}, E \sim 30-35 \mathrm{kPa}\right)$ discussed previously.

From these studies, by covalently introducing PNIPAm side-chains into a PNAGA network, we demonstrate that LCST- and UCST-type phase transitions can be effectively coupled to reinforce the gel both at high and low temperatures. Meanwhile, significant differences can be observed from the association of PNAGA and PNIPAm. Especially, the extensibility of hydrogels strongly increases above the LCST of PNIPAm while it decreases below the UCST of PNAGA. Distinct mechanisms for conformational transition of the two polymers can be taken into account to explain these opposite performances. In the case of PNIPAm, the polymer chains collapse above the LCST through hydrophobic interactions coupled with intra and intermolecular hydrogen bonds. By comparison, the phase separation of PNAGA involves only hydrogen bonds between hydrophilic repeat units. Referring to previous research, it is reported that hydrogen bonds in hydrogels have relatively lower association strength due to the competition of water for binding sites. ${ }^{[31]}$ Consequently, we postulate that in aqueous environment, the hydrogen bonded complexes formed by PNAGA chains can be more easily broken under applied stress. By comparison, the hydrophobic interactions formed within PNIPAm are much more difficult to release in aqueous environment.

In order to emphasize the versatility of our approach, the temperature-responsiveness of hydrogels and consequently their mechanical properties can be modulated by exploiting the phase separation process of thermo-responsive polymers. For instance, it is well known that 
salt addition can modify the hydration level of polymers and then their phase transition temperature. ${ }^{[32,33]}$ Here, turbidity experiments were carried out on PAN gels swollen in $\mathrm{Na} 2 \mathrm{SO} 4$ aqueous solution of different concentrations $(0,0.25$ and $0.5 \mathrm{M})$ (Figure S7 in Supporting Information). The temperature range in which both PNAGA and PNIPAm chains take a coil conformation was reduced from $15{ }^{\circ} \mathrm{C}$ in pure water to $8{ }^{\circ} \mathrm{C}$ in $\mathrm{Na}_{2} \mathrm{SO}_{4}$ at $0.25 \mathrm{M}$. Finally, at $0.5 \mathrm{M}$, the temperature range was shifted to very low temperature and only LCST phase separation of PAN gel was observed.

\section{Conclusions}

In conclusion, both LCST and UCST thermo-responsive phase separation of designed antagonist gels were enabled to trigger large modifications of the mechanical properties and constitute an efficient route for gel toughening. Although the phase separated morphology induced by temperature has been shown to have a strong impact on the mechanical properties, the present study on PAN hydrogels demonstrates that the thermo-reinforcement efficiency also depends on the nature of the associations involved in the phase separation process and consequently on the choice of polymer precursors for the gel synthesis. This is clearly one of the key parameter for the design of mechanically reinforced hydrogels via phase separation mechanism.

\section{Supporting Information}

Materials and Methods, Monomer and hydrogel synthesis, Dynamical Scanning Calorimetry, 1H NMR data, Swelling and Turbidity experiments, Rheology. 
Acknowledgements: The authors gratefully acknowledge the financial support of CNRS, ESPCI, the China Scholarship Council for the Ph.D fellowship funding of H. G. and the Ph.D school of University Pierre and Marie Curie (ED 397, UPMC, Paris, France) for the Ph.D fellowship funding of C. M. The authors also thank G. Ducouret from SIMM for technical advice on performing rheological measurements, A. Michel for inductively coupled plasma analysis and C. Tribet for capillary electrophoresis experiments.

Keywords: hydrogels, isochoric conditions, upper critical solution temperature (UCST), lower critical solution temperature (LCST), mechanical properties

[1] P. Calvert, Adv. Mater. 2009, 21, 743.

[2] W. E. Rudzinski, A. M. Dave, U. H. Vaishanav, S. G. Kumbar, A. R. Kulkarni, T. M. Aminabhavi, Des. Monomers Polym. 2002, 5, 39.

[3] S. Varghese, J. H. Elisseeff, "Hydrogels for musculoskeletal tissue engineering", in Polymers for Regenerative Medicine, C. Werner, J.H. Eisseeff, C. Fischbach, T. Freier, A.J. Garcia, D.J. Mooney, T. Pompe, K. Salchert, S. Varghese, S. Zhang, and X. Zhao, Eds., Springer-Verlag Berlin, Berlin, 2006, p. 95.

[4] K. Haraguchi, T. Takehisa, Adv. Mater. 2002, 14, 1120.

[5] L. Carlsson, S. Rose, D. Hourdet, A. Marcellan, Soft Matter 2010, 6, 3619.

[6] J. P. Gong, Y. Katsuyama, T. Kurokawa, Y. Osada, Adv. Mater. 2003, 15, 1155.

[7] Y. Okumura, K. Ito, Adv. Mater. 2001, 13, 485.

[8] T. Sakai, T. Matsunaga, Y. Yamamoto, C. Ito, R. Yoshida, S. Suzuki, N. Sasaki, M. Shibayama, U. I. Chung, Macromolecules 2008, 41, 5379.

[9] T. L. Sun, T. Kurokawa, S. Kuroda, A. B. Ihsan, T. Akasaki, K. Sato, M. A. Haque, T. Nakajima, J. P. Gong, Nat. Mater. 2013, 12, 932. 
[10] K. Sato, T. Nakajima, T. Hisamatsu, T. Nonoyama, T. Kurokawa, J. P. Gong, Adv. Mater. $2015,27,6990$.

[11] S. Ashraf, H. K. Park, H. Park, S. H. Lee, Macromol. Res. 2016, 24, 297.

[12] L. Klouda, Eur. J. Pharm. Biopharm. 2015, 97, 338.

[13] M. C. Koetting, J. T. Peters, S. D. Steichen, N. A. Peppas, Mat. Sci. Eng. R 2015, 93, 1.

[14] S. R. Van Tomme, G. Storm, W. E. Hennink, Int. J. Pharm. 2008, 355, 1.

[15] M. Shibayama, M. Morimoto, S. Nomura, Macromolecules 1994, 27, 5060.

[16] H. Guo, N. Sanson, D. Hourdet, A. Marcellan, Adv. Mater. 2016, 28, 5857.

[17] H. Guo, C. Mussault, A. Brûlet, A. Marcellan, D. Hourdet, N. Sanson, Macromolecules 2016, 49, 4295.

[18] Y. Liu, X. L. Liu, Y. T. Wu, B. Sun, M. F. Zhu, M. Takafuji, H. Ihara, Chem. Comm. 2010, 46, 430.

[19] M. G. Xia, Y. H. Cheng, Z. Q. Meng, X. Z. Jiang, Z. G. Chen, P. Theato, M. F. Zhu, Macromol. Rapid Comm. 2015, 36, 477.

[20] M. G. Xia, Y. H. Cheng, P. Theato, M. F. Zhu, Macromol. Chem. Phys. 2015, 216, 2230.

[21] B. G. Yang, C. Y. Wang, Y. B. Zhang, L. Ye, Y. F. Qian, Y. Shu, J. M. Wang, J. J. Li, F. L. Yao, Polym. Chem. 2015, 6, 3431.

[22] S. Glatzel, A. Laschewsky, J. F. Lutz, Macromolecules 2011, 44, 413.

[23] J. Seuring, S. Agarwal, Macromol. Rapid Comm. 2012, 33, 1898.

[24] J. Seuring, S. Agarwal, ACS Macro Lett. 2013, 2, 597.

[25] A. Fujihara, N. Shimada, A. Maruyama, K. Ishihara, K. Nakai, S. I. Yusa, Soft Matter $2015,11,5204$.

[26] S. Glatzel, N. Badi, M. Pach, A. Laschewsky, J. F. Lutz, Chem. Comm. 2010, 46, 4517. 
[27] J. Seuring, S. Agarwal, Macromol. Chem. Phys. 2010, 211, 2109.

[28] M. Boustta, P. E. Colombo, S. Lenglet, S. Poujol, M. Vert, J. Control. Release 2014, $174,1$.

[29] J. Seuring, F. M. Bayer, K. Huber, S. Agarwal, Macromolecules 2012, 45, 374.

[30] F. Y. Liu, J. Seuring, S. Agarwal, Macromol. Chem. Phys. 2014, 215, 1466.

[31] E. A. Appel, J. del Barrio, X. J. Loh, O. A. Scherman, Chem. Soc. Rev. 2012, 41, 6195.

[32] F. Liu, J. Seuring, S. Agarwal, J. Polym. Sci. Polym. Chem. 2012, 50, 4920.

[33] Y. Zhang, P. S. Cremer, Ann. Rev. Phys. Chem. 2010, 61, 63. 


\section{Figures}

a

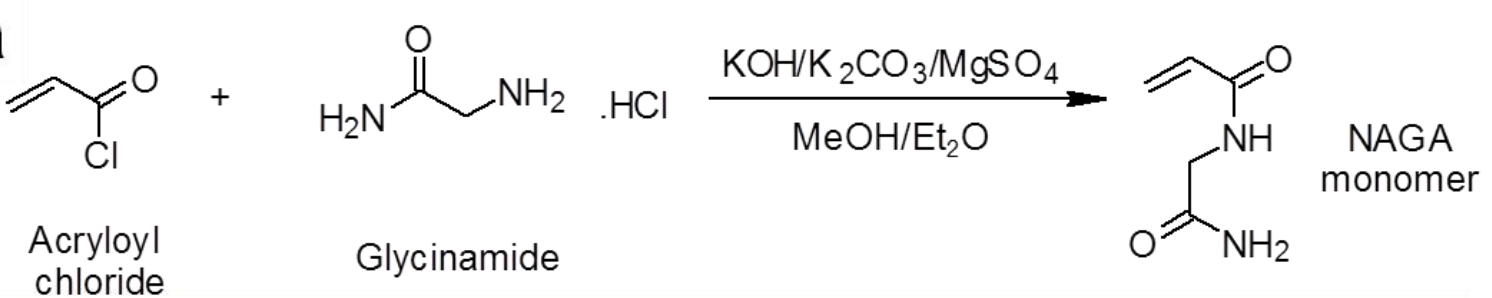

b
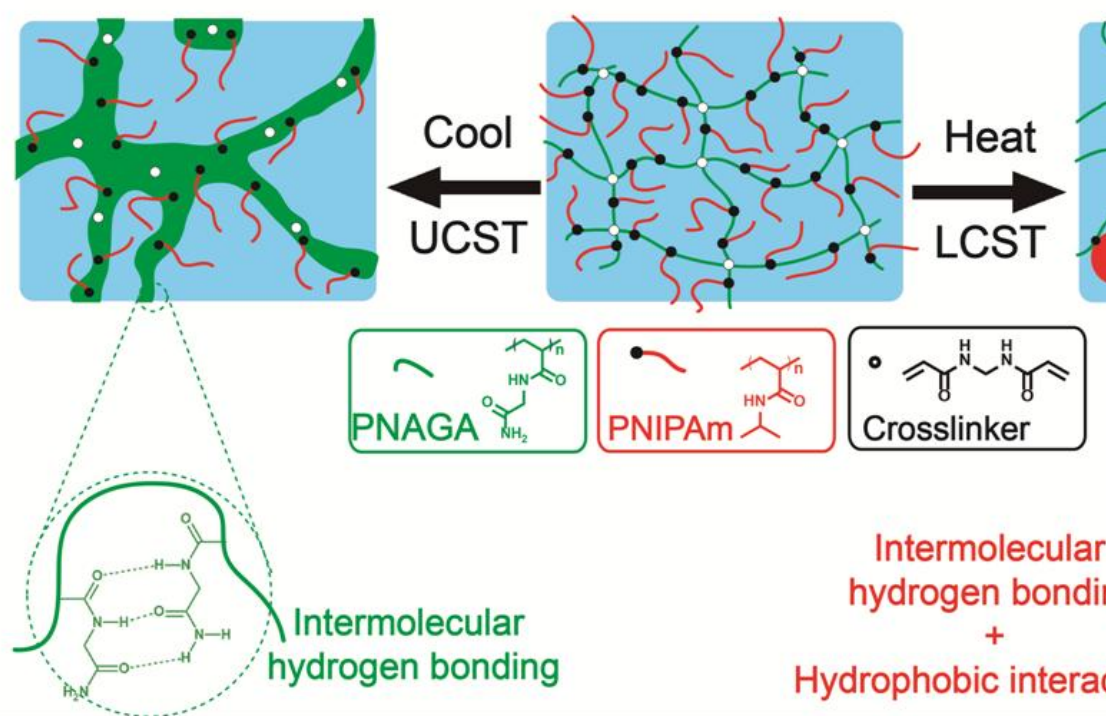

Crosslinker

Intermolecular hydrogen bonding

Hydrophobic interactions

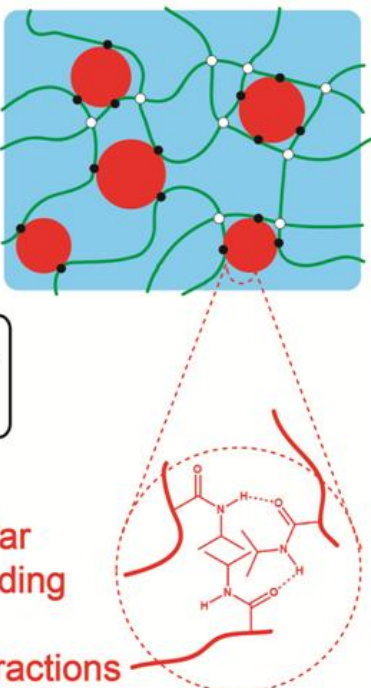

Figure 1. a) Synthesis procedure of NAGA monomer. b) Schematic representation of thermoresponsive self-assembling of PAN hydrogels at low and high temperatures. 

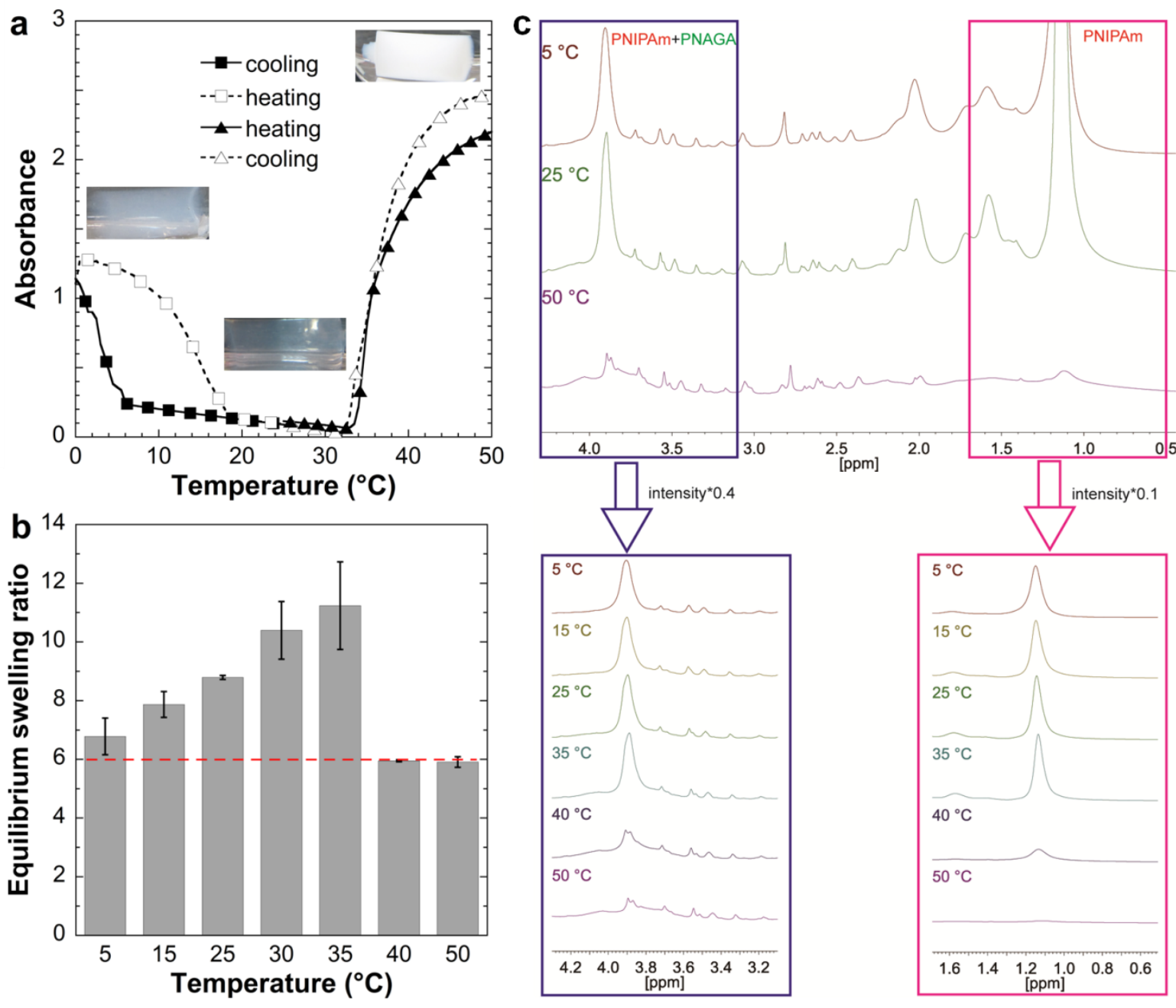

Figure 2. Dual thermo-responsive behavior of PAN hydrogel. a) Turbidity curves as a function of the temperature with a heating/cooling rate of $0.5^{\circ} \mathrm{C} \mathrm{min}^{-1}$. The PAN hydrogel was both cooled and heated from 25 to $0{ }^{\circ} \mathrm{C}$ and from 25 to $50{ }^{\circ} \mathrm{C}$. The insets correspond to their macroscopic appearance at 5,25 and $50{ }^{\circ} \mathrm{C}$. b) Equilibrium swelling ratio, $Q_{\mathrm{e}}$, in pure water at different temperatures. The swelling ratio of the hydrogel at preparation state $Q_{0}=6$ was indicated by the red dotted line. c) Temperature variation of ${ }^{1} \mathrm{H}$ NMR spectra of PAN hydrogel in $\mathrm{D}_{2} \mathrm{O}$ and d) zooms corresponding respectively to the NIPAM and NAGA NMR signals. 


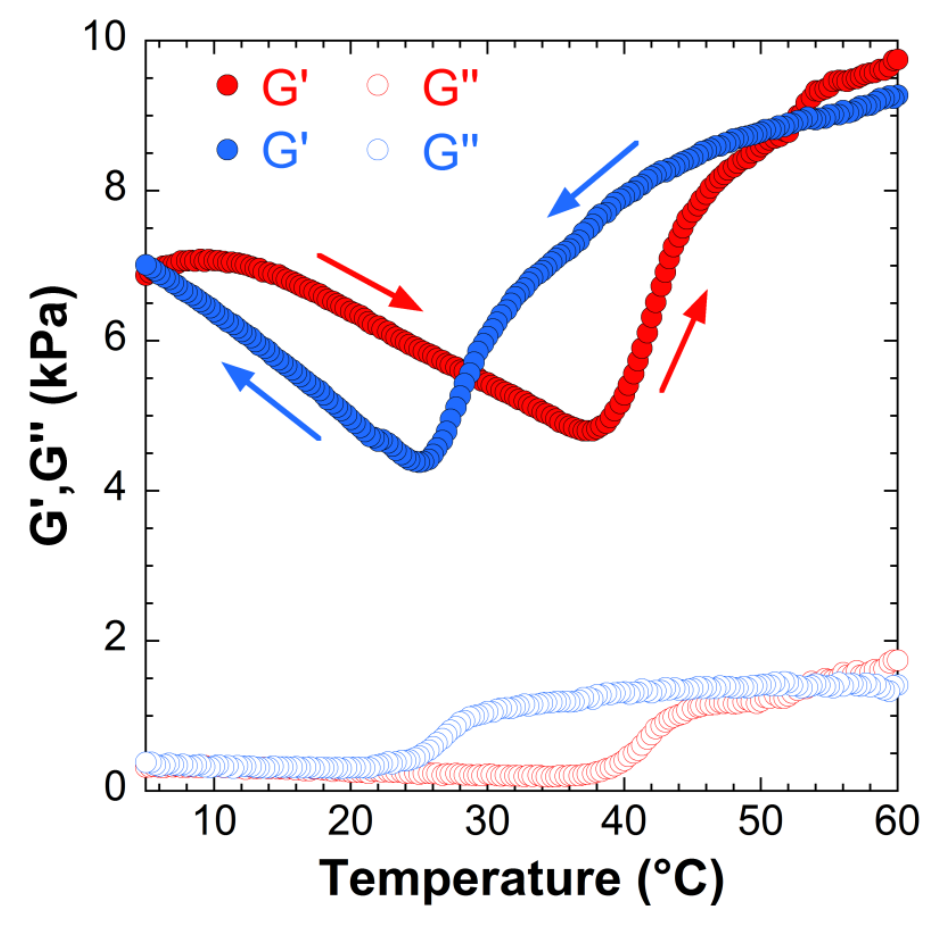

Figure 3. Temperature dependence of viscoelastic properties: elastic modulus, G', (filled symbols) and loss modulus, G", (empty symbols) at $1 \mathrm{~Hz}$ of PAN hydrogel at preparation state with a heating/cooling rate of $2{ }^{\circ} \mathrm{C} \mathrm{min}^{-1}$ (red, upon heating; blue, upon cooling).
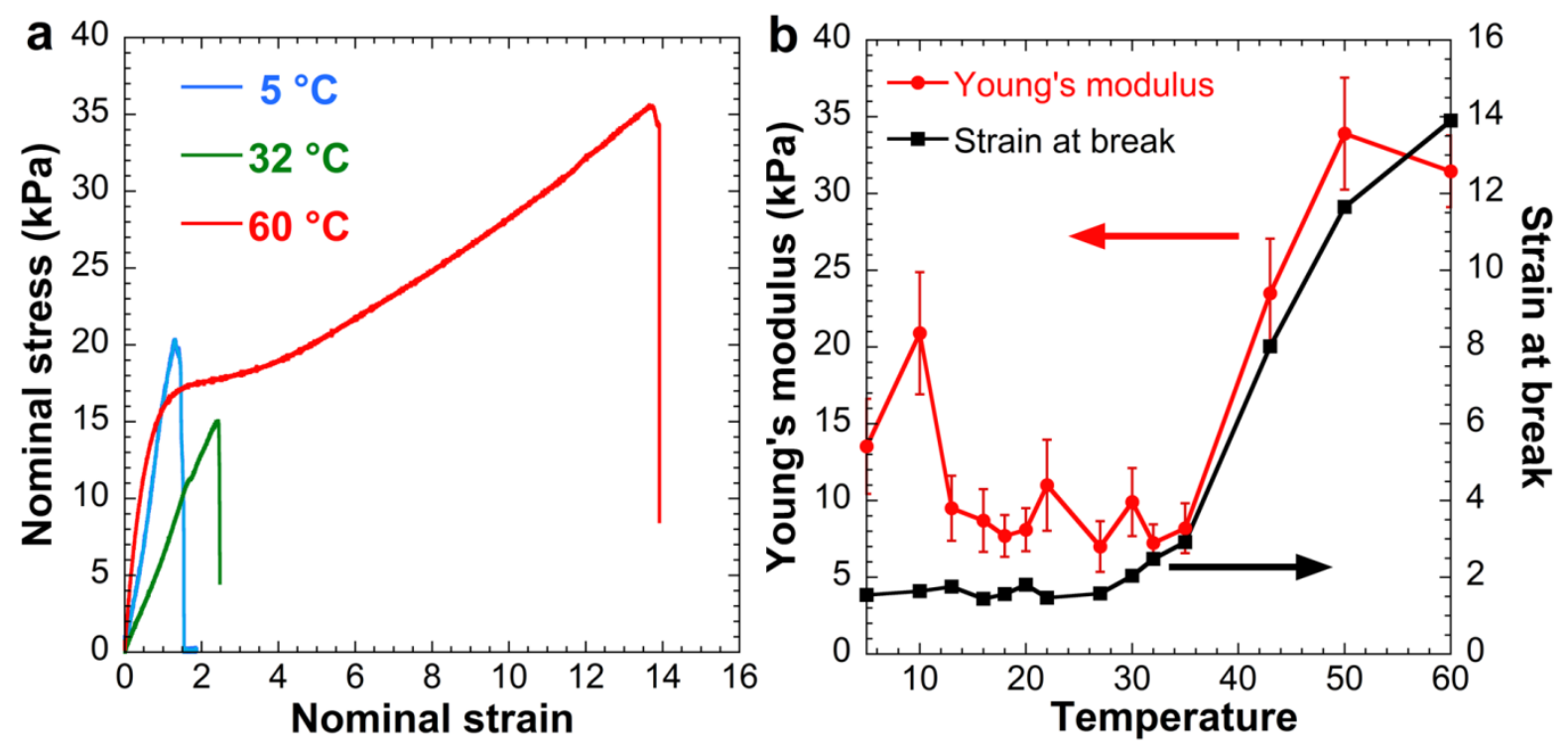

Figure 4. Large strain mechanical behavior of PAN hydrogel (a) Uniaxial tensile stressstrain force curves of PAN gel at 5, 32 and $60{ }^{\circ} \mathrm{C}$. (b) Tensile modulus (red, left axis) and strain at break (black, right axis) of PAN gel at different temperatures (strain rate $=0.06 \mathrm{~s}^{-1}$ ). 
Original chemical hydrogels combining antagonistic upper critical solution temperature (UCST) and lower critical solution temperature (LCST) polymers were elaborated via free radical polymerization. Rheological and mechanical experiments performed under isochoric conditions show a thermo-induced reinforcement both at low and high temperature induced by the formation of phase-separated polymer domains acting as organic reinforcing filler.

H. Guo, C. Mussault, A. Marcellan, D. Hourdet, N. Sanson*

\section{Hydrogels with Dual Thermo-Responsive Mechanical Performance}

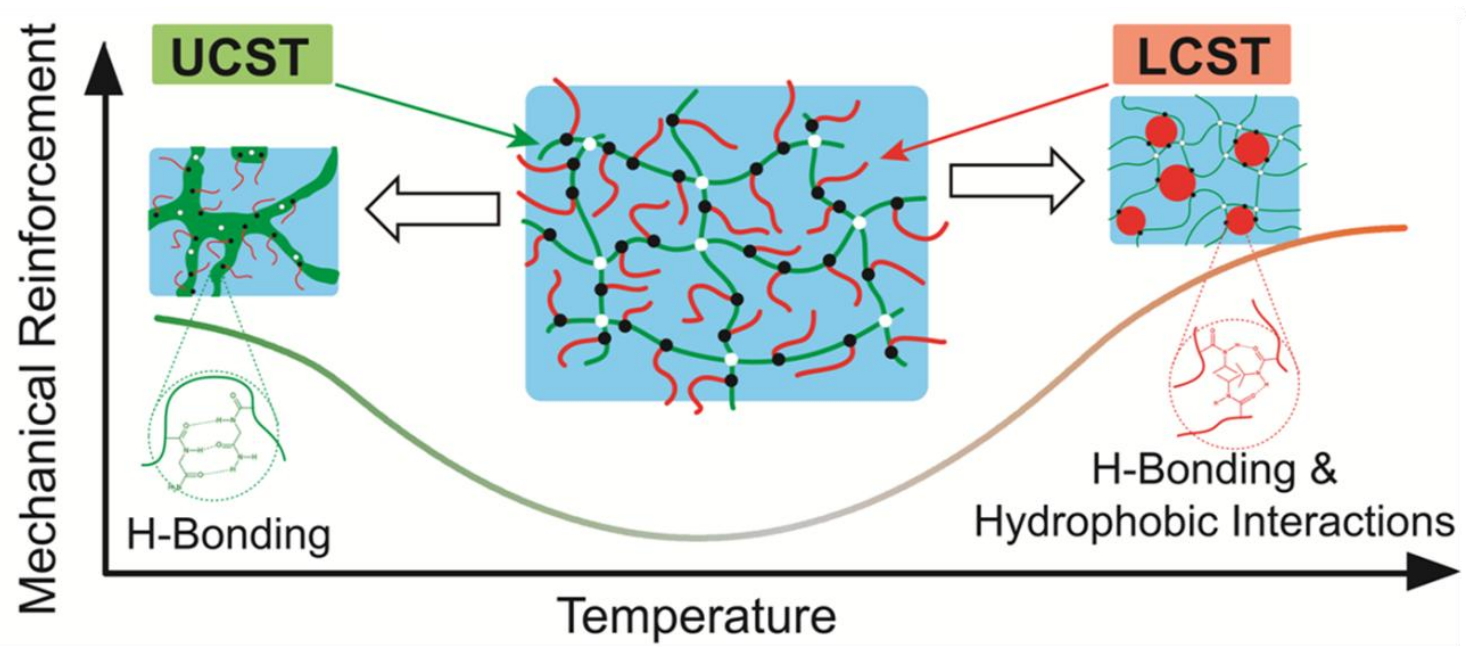


